\title{
Laser spectroscopy of radioactive isotopes: Role and limitations of accurate isotope-shift calculations
}

\author{
B. Cheal, ${ }^{1, *}$ T. E. Cocolios, ${ }^{2, \dagger}$ and S. Fritzsche $\mathrm{e}^{3,4, \ddagger}$ \\ ${ }^{1}$ School of Physics and Astronomy, The University of Manchester, Manchester M13 9PL, United Kingdom \\ ${ }^{2}$ ISOLDE, PH Department, CERN, CH-1211 Geneva 23, Switzerland \\ ${ }^{3}$ GSI Helmholtzzentrum für Schwerionenforschung GmbH, D-64291 Darmstadt, Germany \\ ${ }^{4}$ FIAS Frankfurt Institute for Advanced Studies, D-60438 Frankfurt am Main, Germany
}

(Received 24 July 2012; published 1 October 2012)

\begin{abstract}
Nuclear mean-square charge radii of radioactive isotopes are obtained from isotope-shift measurements using laser spectroscopy. To extract the values of $\delta\left\langle r^{2}\right\rangle$ the atomic field and mass shift factors need to be evaluated. We here summarize the currently available experimental and theoretical techniques for calculating the atomic field and mass shift factors for complex, open-shell atoms, including the $5 s^{2}{ }^{1} S_{0}-4 d 5 p^{1} P_{1}$ and $4 d 5 s{ }^{3} D_{2}-4 d 5 p^{3} P_{1}$ transitions for singly charged yttrium $(Z=39)$.
\end{abstract}

DOI: 10.1103/PhysRevA.86.042501

PACS number(s): 32.30.-r, 21.10.Ft, 21.10.Ky

\section{INTRODUCTION}

Laser-spectroscopic studies are performed on short-lived radioactive nuclei to explore changes in the nuclear size, spin, and moments for isotopes far away from stability [1]. In particular, precision differential changes in the nuclear mean-square charge radius are systematically investigated for long isotopic sequences [2]. These measurements of the optical isotope shifts have long formed the sole probe of such radii [3-5]. Moreover, they are determined independently of any nuclear model.

The changes in mean-square charge radii may be compared directly with the predictions from state-of-the-art nuclear models to test their ability to reproduce macroscopic or microscopic features and refine in some cases their parameters. These models include, for example, the droplet model [6,7], global macroscopic-microscopic calculations [8], the shell model [9,10], self-consistent mean-field models [11] such as a self-consistent relativistic mean-field model [12], Hartree-Fock BCS calculations using a Skyrme interaction [13], Hartree-Fock-Bogoliubov calculations with a Gogny interaction [14-16], or the fermionic molecular dynamics approach [17].

Alternatively, simple nuclear models can be used to extract further observables from the mean-square charge radii, such as the diffuseness of the nuclear surface and the meansquare quadrupole deformation. Unlike the deformation from the nuclear electric quadrupole moment (separately obtained from the hyperfine structure), the mean-square deformation also includes zero-point oscillations. A comparison of these complementary measures of deformation can infer the softness (dynamic nature) or rigidity (static nature) of the deformation, depending on whether the potential energy well as a function of quadrupole deformation is broad or narrow, respectively. However, the mean-square charge radius is also sensitive to higher orders of nuclear deformation. Only with a reliable calibration of the experimental mean-square charge radius $\left\langle r^{2}\right\rangle$ can a meaningful comparison be made with estimates from an

\footnotetext{
*bradley.cheal@manchester.ac.uk

†thomas.elias.cocolios@cern.ch

${ }^{\ddagger}$ S.Fritzsche@gsi.de
}

expression such as

$$
\left\langle r^{2}\right\rangle=\left\langle r^{2}\right\rangle_{\text {sph }}\left(1+\frac{5}{4 \pi}\left\langle\beta_{2}{ }^{2}\right\rangle+\left\langle\beta_{3}{ }^{2}\right\rangle+\cdots\right)+3 \sigma^{2} .
$$

The terms on the right give the volume contribution $\left\langle r^{2}\right\rangle_{\mathrm{sph}}$ for a spherical nucleus of the same volume (given adequately by the droplet model $[6,7])$, the mean-square quadrupole $\left\langle\beta_{2}{ }^{2}\right\rangle$, octupole $\left\langle\beta_{3}{ }^{2}\right\rangle, \ldots$ deformations and the surface diffuseness $3 \sigma^{2}$. Experiments have shown that the diffuseness parameter $\sigma$ is fairly constant across the nuclear chart [3] and it may normally be assumed that these terms cancel if the change in the nuclear charge radius is determined for two isotopes of the same element. Moreover, the quadrupole term $\left\langle\beta_{2}{ }^{2}\right\rangle$ is often assumed to dominate, and is sometimes compared with calculations from nuclear transition rates [18] or substituted for $\left\langle\beta_{2}\right\rangle^{2}$ (obtained from the quadrupole moment) to investigate the dynamic component of the deformation [19].

Aside from studying shape transformations, both the rigidity of the deformation and sharpness of the nuclear surface act also as a probe of the nuclear pairing interaction. Nuclei which posses "magic numbers" of protons or neutrons are particularly stabilized against decay, deformation, and collectivity. For the shell model, therefore, "dips" or "kinks" in the radii are signatures of subshell closure. In addition, the migration of the energy gaps themselves, with a proton or neutron number, is of interest as it gives information on the internucleon interactions. Halo structure [20], parity nonconservation [21], and unitarity tests of the Cabibbo-Kobayashi-Maskawa (CKM) matrix [22] are all phenomena where $\delta\left\langle r^{2}\right\rangle$ plays a key role.

For a reliable and detailed analysis of the nuclear properties, accurate atomic calculations are often needed to separate the electronic response from the nuclear response in the shifts in the resonance signal of the laser transitions. Although progress has been made in determining the mass- and field-shift contributions of the electrons to the overall isotope shifts, limitations arise from the shell structure of the atoms or ions used for laser spectroscopy as well as from the incomplete treatment of the relativistic and correlation contributions of the mass shift operator. While progress has been made for light elements such as beryllium [23] where only a few electrons are present and full ab initio calculations are performed, such an approach is not feasible for medium and heavy elements, 
especially if open $p$ and $d$ shells are involved in the atomic transitions.

In this work, we review the present status of isotope shift (IS) calculations and how they are utilized to extract nuclear properties. Examples are given for various recent and ongoing experiments for mid- and high- $Z$ isotopic chains. With these examples, the importance and necessity of accurate atomic calculations will be highlighted. The results and implications of such computations for the structure of the nuclei have been detailed elsewhere. Finally, a few conclusions are drawn about which atomic transition and electronic structures are favorable for future calculations and experiments. New cases, both on the experimental and theoretical sides, are also proposed.

\section{OBSERVABLES}

The nuclear mean-square charge radius is defined in terms of the nuclear charge distribution $\rho(\mathbf{r})$ as

$$
\left\langle r^{2}\right\rangle=\frac{\int \rho(\mathbf{r}) r^{2} d V}{\int \rho(\mathbf{r}) d V} .
$$

Although nuclei produced from the nuclear reactions will have a distribution in $Z$ as well as $N$ (even when a particular mass number $A=Z+N$ is selected), the nature of atomic transitions and the practicalities in producing the laser wavelengths mean that only one element is studied during a particular experiment. Changes in resonance frequency between isotopes is much easier to determine with precision than a measurement of absolute transition frequencies and nuclear parameters more readily extracted.

The changes in mean-square charge radii $\delta\left\langle r^{2}\right\rangle^{A, A^{\prime}}=$ $\left\langle r^{2}\right\rangle^{A^{\prime}}-\left\langle r^{2}\right\rangle^{A}$ between isotopes $A$ and $A^{\prime}$ are determined from IS measurements $\delta v^{A, A^{\prime}}=v^{A^{\prime}}-v^{A}$ via

$$
\delta v_{i}^{A, A^{\prime}}=M_{i} \frac{A^{\prime}-A}{A A^{\prime}}+F_{i} \delta\left\langle r^{2}\right\rangle^{A, A^{\prime}},
$$

where $M_{i}$ and $F_{i}$ are the mass shift and field shift factors for the considered atomic (or ionic) transition $i$ and accurate values for the isotopic masses are substituted in place of the mass numbers $A$ and $A^{\prime}$. For heavier nuclei, $\delta\left\langle r^{2}\right\rangle$ is replaced with the Seltzer moment [24]

$$
\lambda^{A, A^{\prime}}=\delta\left\langle r^{2}\right\rangle^{A A^{\prime}}+b_{1} \delta\left\langle r^{4}\right\rangle^{A A^{\prime}}+b_{2} \delta\left\langle r^{6}\right\rangle^{A A^{\prime}} \cdots .
$$

Terms of higher order than $\delta\left\langle r^{2}\right\rangle$ contribute only a few percent even in heavy nuclei, and the approximation

$$
\lambda^{A, A^{\prime}}=\delta\left\langle r^{2}\right\rangle^{A A^{\prime}}\left(1+b_{1} \frac{\delta\left\langle r^{4}\right\rangle^{A A^{\prime}}}{\delta\left\langle r^{2}\right\rangle^{A A^{\prime}}}+\cdots\right)=K(Z) \delta\left\langle r^{2}\right\rangle^{A A^{\prime}}
$$

is often used, where $K(Z)$ has been tabulated and is close to unity [25].

From an atomic physics point of view, the two factors $M_{i}$ and $F_{i}$ need to be determined for each transition separately. These two factors are assumed to be constant for an isotopic chain and, thus, enable one to extract $\delta\left\langle r^{2}\right\rangle$ without any dependence on the nuclear model. For many elements, three or more isotopes are naturally abundant, which allows (at least) two values of $\delta\left\langle r^{2}\right\rangle$ to be measured using nonoptical techniques. These values can be used in conjunction with IS measurements to calibrate the two factors and therefore extract $\delta\left\langle r^{2}\right\rangle$ from IS measurements for all isotopes, whether they are naturally occurring, or radioactive and short-lived, with only small fluxes available from a reaction.

Although the atomic factors are transition dependent, if they are known for one transition they can also be determined for other transitions using the King plot technique [1,26]. Multiplying Eq. (3), for example, by a modification factor $A A^{\prime} /\left(A^{\prime}-A\right)$ gives

$$
\frac{A A^{\prime}}{A^{\prime}-A} \delta v_{i}^{A A^{\prime}}=M_{i}+F_{i} \frac{A A^{\prime}}{A^{\prime}-A} \delta\left\langle r^{2}\right\rangle^{A A^{\prime}} .
$$

For two transitions $i$ and $j$, this allows $\delta\left\langle r^{2}\right\rangle$ to be eliminated and gives rise to the linear regression line

$$
\left(\frac{A A^{\prime}}{A^{\prime}-A} \delta v_{j}^{A, A^{\prime}}\right)=\frac{F_{j}}{F_{i}}\left(\frac{A A^{\prime}}{A^{\prime}-A} \delta v_{i}^{A, A^{\prime}}\right)+M_{j}-\frac{F_{j}}{F_{i}} M_{i} .
$$

Therefore, if IS data for two transitions are modified by the factor $A A^{\prime} /\left(A-A^{\prime}\right)$, the plot of such values will be linear with a gradient of $F_{j} / F_{i}$, and intercept $M_{j}-\left(F_{j} / F_{i}\right) M_{i}$.

The transitions chosen for experimental study will be selected on grounds such as spectroscopic efficiency to measure the most exotic (and weakly produced) isotopes. However, these may not be the most convenient lines for which to consider reliable theoretical computations of their atomic factors. For a (second) transition which is nonoptimal from an experimental point of view, a selected remeasurement of a few isotope shifts can be made, but will be limited to the more intensely produced isotopes.

Using the King plot technique, one can then proceed in two ways. First, this technique enables the atomic factors for the primary (spectroscopically efficient) transition to be determined from those calculated with relative ease for a purer transition. Only two isotope shifts would need to be remeasured using the second transition (or two existing $\delta\left\langle r^{2}\right\rangle$ values if available). Alternatively, the King plot can be used as a consistency check of the theoretical computations of $F$ and $M$ since the King plot provides a definite relationship between the factors for one transition and those of another. If, for example, the ratio of the two ab initio calculated $F$ factors is close to the (experimentally determined) gradient of the King plot, then confidence in these values is increased.

\section{EXPERIMENTAL TECHNIQUES}

Of the available techniques in the study of charge radii, laser spectroscopy of optical isotope shifts is versatile and has been extensively applied to small fluxes of short-lived radioactive nuclei [1]. These nuclei are produced at radioactive ion beam facilities and the spectroscopy is performed in situ. Two common forms exist: the "collinear beams" method [17, $22,27,28]$, which achieves the highest precision, and the "insource" method [29,30] which is currently applicable to lower atom fluxes. One or the other is generally used and these are discussed below, with a schematic overview in Fig. 1. Variations on those techniques can be used in specific cases (e.g., Refs. [31-34]). 


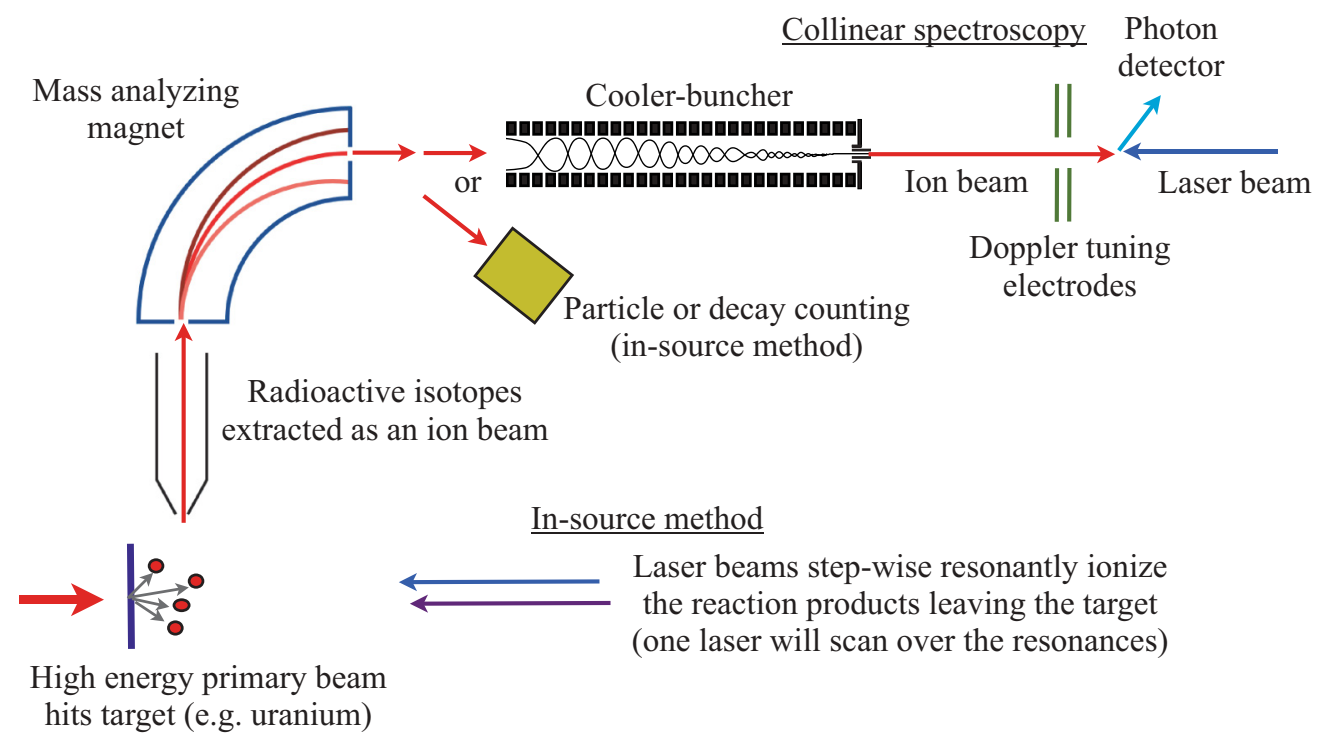

FIG. 1. (Color online) Schematic view of the two common forms of laser spectroscopy of radioactive isotopes. One of these two techniques is usually selected, although during collinear experiments, the lasers used for in-source spectroscopy are often used to selectively enhance the ionisation of the reaction products for the element of interest [35]. In this case, the lasers are not scanned but run in a broadband mode.

\section{A. Collinear laser spectroscopy}

At radioactive isotope production facilities, nuclear reactions are triggered when a high-energy primary beam hits a piece of target material to cause fission, fusion, spallation, and/or any additional mechanism. The isotopes produced diffuse or recoil from the target material into, or are guided to an ion source, where ionization to a singly charged state takes place. The electrodes are then used to form a beam of these ions, which are accelerated away to energies of typically 30 to $60 \mathrm{keV}$. The isotopes, which may have half-lives of only a few milliseconds, are then mass selected in-flight and delivered to the high-resolution laser spectroscopy station.

The laser and atom beams are overlapped in a collinear or anticollinear geometry to maximize the interaction length and therefore the probability of excitation. Photomultiplier tubes (PMTs) are mounted at $90^{\circ}$ to the beams and plane of polarization, to detect fluorescence photons. Doppler broadening of the hyperfine resonances results from the velocity spread $\delta v$ of the ions in the forward direction, caused by the kinetic energy spread $\delta E$ in the ionization region. However, from the classical kinetic energy formula $\delta E=m v \delta v$, it can be seen that the use of fast $(\sim 30 \mathrm{keV})$ beams suppresses this velocity spread since the energy spread remains constant. A tuning potential is applied to the point of interaction to Doppler shift the observed frequency over the resonances, or to an alkali vapor cell immediately upstream of the interaction region in the cases where neutral atoms are investigated.

In the last decade, gas-filled radio-frequency quadrupoles, known as ion beam coolers, have been used to capture and reaccelerate the mass-filtered ion beam en route to the laser spectroscopy station [18]. These devices cool the ions, reducing the energy spread through collisions with a helium buffer gas. More importantly, ion coolers have been used to accumulate and trap the ions for up to $500 \mathrm{~ms}$ and release them in bunches of a few microseconds temporal length. This maintains the intensity of the resonant fluorescence signal, while enabling suppression of the photon background from the continuous scattering of laser light, by the ratio of these accumulation and release times (approximately four orders of magnitude). More recently, optical pumping has been performed inside the ion cooler [27], enabling metastable states of interest to be populated. This has permitted collinear laser spectroscopy to be performed from these states in preference to the ground state, where required.

Using these techniques, resonance widths are of the same order of magnitude as the natural linewidth, and the spectroscopy on ion fluxes of 100/s has been routinely performed [36].

\section{B. In-source laser spectroscopy}

Conversely to the aforementioned in-flight technique, in-source laser spectroscopy is performed directly at the secondary beam production level. By sending a set of laser beams directly into the ion source, a valence electron may be excited across the atomic structure until it reaches beyond the ionization potential. This ionization technique is regularly used in two types of radioactive ion beam facilities: thick-target hot-cavity facilities, where the reaction products are stopped in the material and diffuse from within, and thin-foil-target gas-catcher facilities, where products recoil directly into a gas stopping volume. In-source spectroscopy has proven efficient and selective at both hot-cavity facilities [37-40] and gascatcher facilities [41-43].

The spectroscopy is performed by monitoring the ion beam intensity as a function of the frequency of one of the resonant transitions. The ion beam intensity may be recorded by counting ions or observing the radioactive decay of the mass-separated beams. Either technique is very sensitive and the radioactive decay can be used to identify long-lived nuclear isomers from the ground state [44]. This increased sensitivity allows the study of much more exotic cases than the collinear 
technique. Isotopes with production rates down to 6 ions/s at a gas-catcher facility [29] and less than 1 ion/s at a hot-cavity facility $[30,45]$ have been reached.

The limit of the technique is, however, the large energy broadening due to the environmental conditions inside the ion source. At hot-cavity facilities, the high temperature of the ion source $\left(\sim 2000^{\circ} \mathrm{C}\right)$ induces a large velocity spread, resulting in a typical Doppler broadening of $\sim 4 \mathrm{GHz}$; at gas-catcher facilities, collisions with buffer gas atoms and molecules result in a pressure broadening of a similar magnitude [46].

As a result of this large broadening, only limited information can be extracted from the data. The magnetic dipole moment may be extracted if the hyperfine parameter is large enough, as in copper [29]. In the case of $\delta\left\langle r^{2}\right\rangle$, as seen in Eq. (3), the mass shift diminishes with increasing $A$ while the field shift factor $F_{i}$ increases with increasing $Z$. As a consequence, heavy isotopes have large isotope shifts which can be accessed with in-source techniques [30,45,47-49].

There exists an intermediate concept between the in-source ionization and in-flight fluorescence techniques in the collinear resonant ionization spectroscopy (CRIS) technique [50,51]. By ionizing the beam of interest in-flight, it is possible find a compromise between the high resolution obtained upon accelerating the beam and from the high efficiency from the ion detection technique. This technique is currently under development at CERN ISOLDE [52].

\section{Nonoptical methods}

Nuclear charge radii have also been measured using nonoptical techniques: primarily $K$ x-ray spectroscopy (via the Seltzer moment $\lambda$ ), muonic atom spectroscopy (Barrett radii), and electron scattering [the nuclear density distribution, $\rho(r)$ ] [53]. Measurements using the nonoptical methods generally require preparation of relatively large (quasistable) samples. It has therefore not been possible to apply such techniques to radioactive ion beam production. Nevertheless, a combined analysis of data from these techniques can provide absolute charge radii data for the longest lived nuclei [54,55]. The total $\left\langle r^{2}\right\rangle$ can then be determined for any isotope using these values in combination with the differential $\delta\left\langle r^{2}\right\rangle$ values from optical spectroscopy.

Where nonoptical measurements can be made for three isotopes of an element (i.e., values of $\delta\left\langle r^{2}\right\rangle$ are available for two pairs of isotopes), the two atomic factors can also be determined using a King plot analysis similar to the one described in Sec. II. Values of $\delta\left\langle r^{2}\right\rangle$ can be used in place of the IS values for one transition. They can then be considered as IS values for a hypothetical transition where $F=1$ and $M=0$. However, many elements have fewer than three stable isotopes, and theoretical calculations must be relied upon.

\section{THEORY AND COMPUTATIONS}

Various methods have been developed over the years to evaluate isotope shifts and parameters for different elements and electronic transitions; aside from some early semiempirical estimates, systematic IS calculations have become available only recently and showed how sensitive these shifts may depend on the electronic structure of atoms or ions. Here, we shall not recall the basic IS theory in detail, which has been presented in the literature [56-58]. Instead, we will focus on the difficulties and limitations that arise from the complex shell structure of medium and heavy elements, and especially for atoms with open $p$ and $d$ shells.

\section{A. Parametrization of isotope shifts}

The parametrization in Eq. (3) assumes that the IS in the transition frequency of an atomic line $\delta v_{i}^{A, A^{\prime}}$ depends on the masses $A$ and $A^{\prime}$ of a given pair of isotopes and their change in the mean-square charge radius $\delta\left\langle r^{2}\right\rangle^{A, A^{\prime}}$, but not on further details of the nuclear potential as it appears in the electronic structure calculations. For many (chains of) isotopes, indeed, this assumption appears to be well fulfilled and, thus, supports such a separation of the nuclear properties from the electronic response, up to some reasonable but not further specified nuclear model. With these assumptions in mind, Eq. (3) enables one to capture the response of the electrons upon changes in the recoil of the nucleus and the nuclear potential for a whole chain of isotopes in just two parameters, namely the mass-shift parameter $M$ and field-shift parameters $F$, respectively. The mass shift of the atomic energy levels arises from the recoil of the nucleus (i.e., the energy-momentum conservation of the composite system "nucleus + electrons"), and is usually separated into two parts: The normal mass shift $M_{\mathrm{NMS}} \approx v_{\infty} \frac{m_{e}}{m_{n}}$, as obtained from the reduced electron mass (due to its mass ratio with regard to the mass of the nucleon $m_{n}=1820 m_{e}$ ) as well as the specific mass shift $M_{\mathrm{SMS}}$ that reflects the correlations of the momenta $\left\{\vec{p}_{i}, i=1, \ldots, N_{e}\right\}$ of the electrons. The field shift of atomic levels and transition frequencies, in contrast, originates from changes in the nuclear charge distribution and volume.

For optical transitions, the IS is obtained as the difference of two shifts for the upper and lower atomic levels. While the individual shifts of the atomic levels can be quite large, in general, they partly cancel each other out and make it necessary to calculate the various contributions with sufficient accuracy to derive useful information about the nucleus. We note, however, that the parametrization in Eq. (3) and its factorization into some electronic $\times$ nuclear property is an approximation which may break down if the nuclear radii and further moments of the charge and magnetization distribution are known with enough accuracy.

\section{B. Semi-empirical estimates versus many-body calculations}

Many of the earlier field-shift studies were focused on the $n s-n p$ optical transitions of atoms and ions with either an alkali-metal or alkaline-earth-metal valence configuration. For these shell structures, the field-shift parameter $F_{i}$ has often been parametrized as $[25,53]$

$$
F_{i}=\frac{\pi a_{o}}{Z} \Delta\left|\psi_{n s}(0)\right|_{\mathrm{nr}}^{2} f_{i}(Z)
$$

where $\Delta\left|\psi_{n s}(0)\right|_{\text {nr }}^{2}$ describes the difference of the total nonrelativistic density distribution of the active $n s$ electron for a pair of isotopes with $A, A+1$, taken for a point nucleus, and where $f_{i}(Z)$ describes the relativistic correction to $F_{i}$ for a finite model of nuclear charge distribution. Although this parametrization is simple and has been found reasonably reliable [56], it neglects the screening of the $n s$ electron in 
proximity to the nucleus by the other electrons, the changes of the nuclear potential upon the excited $n p$ electron as well as correlation effects. Moreover, such a simple parametrization cannot be derived so easily for other transitions that do not include a $n s{ }^{2} S_{1 / 2}$ or $n s^{2}{ }^{1} S_{0}$ ground level of either an alkali-metal-like or alkaline-earth-metal shell structure.

In the laser spectroscopy of most medium and heavy elements, in fact, optical transitions with a sufficiently strong (E1) absorption rate are considered that do not include the ground state necessarily but can be explored by lasers as available already (in the laboratory, for instance). For a thorough treatment of both the mass and field shifts, it then appears more appropriate to start from an ab initio description of the IS that is performed independently for a few selected isotopes, and by including all important mass and field-shift contributions. In such a theoretical treatment of the IS, $M$ and $F$ are simply used to parametrize the shifts, analog as in experiments, and by applying some reasonable nuclear charge distribution in the computations. The variation of the $M$ and $F$ parameters, obtained for different sets of three isotopes, are interpreted first of all as numerical uncertainties due to missing correlation and relativistic effects as well as with regard to the overall stability of the computations. Only if the $M$ and $F$ are not much affected by the details of the nuclear model, taken, for instance, as a Fermi-distributed charge, are these parameters deemed useful and can be exploited in the extraction of nuclear observables (in a seemingly model-independent way).

\section{Atomic mass-shift operators}

For an atom with mass $M_{a}$, the mass shift of an atomic level is caused by the recoil motion of the nucleus in the combined system "nucleus + electrons" and goes as the first order in the mass ratio $\left(m_{e} / M_{a}\right)$, described by the Hamiltonian [57]

$H_{\mathrm{MS}}=\frac{1}{2 M_{a}} \sum_{i j}^{N_{e}}\left[\mathbf{p}_{i} \cdot \mathbf{p}_{j}-\frac{\alpha Z}{r_{i}}\left(\boldsymbol{\alpha}_{i}+\frac{\left(\boldsymbol{\alpha}_{i} \cdot \mathbf{r}_{i}\right) \mathbf{r}_{i}}{r_{i}^{2}}\right) \cdot \mathbf{p}_{j}\right]$.

Typically, this Hamiltonian is separated into two parts, the normal mass shift and the specific mass shift, $H_{\mathrm{MS}}=$ $H_{\mathrm{NMS}}+H_{\mathrm{SMS}}$ to distinguish the one-electron terms, due to the reduced electron mass, from the two-electron contributions of the electronic momenta. These two parts of the mass-shift Hamiltonian then read

$$
\begin{gathered}
H_{\mathrm{NMS}}=\frac{1}{2 M_{a}} \sum_{i}^{N_{e}}\left(\mathbf{p}_{i}^{2}-\frac{\alpha Z}{r_{i}} \boldsymbol{\alpha}_{i} \cdot \mathbf{p}_{i}-\frac{\alpha Z}{r_{i}}\left(\boldsymbol{\alpha}_{i} \cdot \mathbf{C}_{i}^{1}\right) \mathbf{C}_{i}^{1} \cdot \mathbf{p}_{i}\right) \\
H_{\mathrm{SMS}}=\frac{1}{M_{a}} \sum_{i<j}^{N_{e}}\left(\mathbf{p}_{i} \cdot \mathbf{p}_{j}-\frac{\alpha Z}{r_{i}} \boldsymbol{\alpha}_{i} \cdot \mathbf{p}_{j}\right. \\
\left.-\frac{\alpha Z}{r_{i}}\left(\boldsymbol{\alpha}_{i} \cdot \mathbf{C}_{i}^{1}\right) \mathbf{C}_{i}^{1} \cdot \mathbf{p}_{j}\right)
\end{gathered}
$$

and their further decomposition into a tensorial form was worked out recently by Gaidamauskas et al. [59]. An analog decomposition applies also for the mass-shift parameter $M=$ $M_{\mathrm{NMS}}+M_{\mathrm{SMS}}$. For low- $Z$ atoms and ions $(\alpha Z \ll 1)$, only the first term in Eqs. (10) and (11) are important, while the other terms typically become significant for heavy elements, and especially if multiply or highly charged ions are considered [57]. However, at present, very little is known about the importance of these relativistic terms if optical transitions are considered for medium and heavy atoms and ions with a low charge state.

In the computations reported in this article, the main difficulties arise from a proper treatment of the electron-electron interaction and the (large) uncertainties due to the missing relativistic and correlation contributions. Large uncertainties in the calculated $M$ and $F$ parameters are then not uncommon for the transitions of interest. As such, the mass-shift operators in Eqs. (10) and (11) have been restricted in the calculations below to their nonrelativistic part as obtained for $\alpha Z \rightarrow 0$. For the normal mass shift, moreover, we simply use $M_{\mathrm{NMS}}=$ $v_{\infty} \frac{m_{e}}{m_{n}}$ in all cases below.

\section{Ab initio isotope-shift calculations for open-shell atoms}

Different many-body techniques have been designed to calculate (or, at least, estimate) the IS of atomic levels and transitions. For atoms and ions with a complex shell structure, the multiconfiguration Dirac-Fock (MCDF) method has been found to be a very versatile tool as it enables one to describe atoms with two and more open shells in quite a similar manner as closed-shell systems. A thorough treatment of the MCDF method may already be found in the literature [65]. Its use applied to a large number of atomic level calculations as well as for predicting transitions and ionization properties has also been reported in Refs. [66,67].

In the MCDF method, an atomic state is approximated by a linear combination of the so-called configuration state functions (CSF) of the same symmetry

$$
\psi_{\alpha}(P J M)=\sum_{r=1}^{n_{c}} c_{r}(\alpha)\left|\gamma_{r} P J M\right\rangle,
$$

where $n_{c}$ is the number of CSF and $\left\{c_{r}(\alpha)\right\}$ denotes the representation of the atomic state in this basis. In most standard computations, the CSF $\left|\gamma_{r} P J M\right\rangle$ are constructed as antisymmetrized products of a common set of orthonormal orbitals and are optimized together on the basis of the DiracCoulomb Hamiltonian. Relativistic effects due to the Breit interaction are then added to the representation $\left\{c_{r}(\alpha)\right\}$ by diagonalizing the Dirac-Coulomb-Breit Hamiltonian matrix $[68,69]$. While the dominant QED corrections can at least be estimated within this method, they are typically negligible for optical transitions of medium and heavy elements. In addition, the specific mass-shift operator is taken into account into the Hamiltonian matrix, while the different charge distribution of the isotopes, related to the field shift, are considered implicitly by means of an extended nucleus. In the computations below, we applied a two-parameter Fermi distribution

$$
\rho(r)=\frac{\rho_{o}}{1+e^{(r-c) / a}},
$$

where $c$ denotes the "half-charge density" radius and $a$ the skin thickness. Since we aim for a single field-shift parameter $F$ for a whole chain of isotopes, a spherical symmetric nucleus has been assumed for all isotopes, independent of 
their natural occurrence, and the Fermi parameters were taken as implemented in GRASP92 [68].

For atoms with a complex shell structure, the major difficulties arise from unaccounted correlation contributions, as is typical for most many-body procedures. In the MCDF method, these correlations are taken into account by incorporating systematically single, double, and possibly further replacements of electrons from bound orbitals into some active set of virtual orbitals. Using such a "restricted active space" procedure, however, the size of the wave-function expansion $n_{c}$ in Eq. (12) often increases rapidly and makes it necessary to restrict the size of the expansions. In the past, such an approach has been utilized mainly to explore the IS of light elements [70] and simple valence shell structures [71], while little work has been done for atoms and ions with complex spectra, say, with more than two electrons outside of closed shells.

Contrary to the semi-empirical parametrization in Eq. (8), in which the field-shift parameter is obtained for a pair of neighboring isotopes, we applied first-order perturbation theory for a series of isotopes and calculated the corresponding IS of the upper and lower levels involved in the transition explicitly by diagonalizing the full Hamiltonian matrix including the specific mass shift and the extended nucleus. A common set of radial orbitals, as generated for some stable isotope, is typically sufficient because of the overall small shifts of the atomic levels, compared, for instance, to the fine structure of the levels. For any three selected isotopes from the few calculated, the mass-shift $M$ and field-shift parameters $F$ are then obtained by solving Eq. (3). Although this procedure gives rise to variations in the calculated $M$ and $F$ values (as calculated for different sets of three isotopes), it nicely reflects the deficiencies in the model and the stability of the computations. For well-correlated wave functions, indeed, the variations are much smaller than the changes of these parameters for different computational models and, hence, the average of the $M$ and $F$ for different sets are used for the parametrization in Eq. (3). This behavior of the IS parameters also confirms our conclusions that the major difficulties in obtaining accurate mass-shift and field-shift parameters arise from the treatment of the electron-electron correlations.

\section{E. Systematically enlarged multiconfiguration Dirac-Fock wave computations}

To support ongoing experiments at the IGISOL facility in Jyväskylä, the ISOLDE facility at CERN, and elsewhere, MCDF calculations have been performed for various elements during recent years, from the rather light $\mathrm{Sc}^{+}$ions with an open $3 d$ shell up to Po atoms with a half-filled $6 p$ shell (cf. Table I). These calculations showed the sensitivity of the IS parameters with regard to the shell structure and different correlation contributions in the expansion of the many-electron wave functions. In the MCDF model, a series of computations is typically carried out for each atomic transition of interest, in which the size of the wave-function expansion is systematically enlarged and the $M$ and $F$ parameters are monitored with regard to different classes of correlations. In most of the reported cases, however, we were not able so far to demonstrate real convergence while the size of the wave functions is increased and further computations are needed to learn more about the various shell structures and classes of correlations that are important for a proper description.

Apart from the optical transitions of elements that have been investigated previously (see Table I for examples and

TABLE I. Transitions used in the laser spectroscopy of radioactive isotopes. Apart from the wavelength and wave numbers of these transitions, we here display estimates for the (total) mass and field-shift parameters $M$ and $F$, as obtained in different case studies during recent years. The values in parentheses show the estimated accuracy (in percent) of the calculated IS parameters. Many other elements are without any form of calibration of the atomic factors. Some transitions which are expected to be studied experimentally in the near future (and require atomic factor calculations) are also included.

\begin{tabular}{|c|c|c|c|c|c|c|c|}
\hline \multicolumn{2}{|c|}{ Element } & \multirow{2}{*}{$\begin{array}{r}\text { Transition } \\
3 d 4 s^{3} D_{2}-3 d 4 p^{3} F_{3}\end{array}$} & \multirow{2}{*}{$\begin{array}{c}\begin{array}{c}\text { Wavelength } \\
\text { [nm] }\end{array} \\
363.2\end{array}$} & \multirow{2}{*}{$\begin{array}{c}\begin{array}{c}\text { Wave number } \\
{\left[\mathrm{cm}^{-1}\right]}\end{array} \\
27534.7\end{array}$} & \multirow{2}{*}{$\left.\begin{array}{c}M \\
{[\mathrm{GHz} \mathrm{amu}]}\end{array}\right]$} & \multirow{2}{*}{$\begin{array}{c}F \\
{\left[\mathrm{MHz} / \mathrm{fm}^{2}\right]} \\
-355(15 \%)\end{array}$} & \multirow{2}{*}{$\begin{array}{c}\begin{array}{c}\text { Method } \\
\text { \& Ref. }\end{array} \\
\text { MCDF [10] }\end{array}$} \\
\hline $\mathrm{Sc}^{+}$ & $(Z=21)$ & & & & & & \\
\hline $\mathrm{Mn}^{+}$ & $(Z=25)$ & $3 d^{5} 4 s^{5} S_{2}-3 d^{5} 4 p^{5} P_{3}$ & 295.0 & 33897.5 & $-572(15 \%)$ & $852(10 \%)$ & MCDF [60] \\
\hline Mn & $(Z=25)$ & $3 d^{5} 4 s^{2}{ }^{6} S_{5 / 2}-3 d^{5} 4 s 4 p^{6} P_{3 / 2}$ & 280.2 & 35690.0 & - & - & - \\
\hline $\mathrm{Cu}$ & $(Z=29)$ & $3 d^{10} 4 s^{2} S_{1 / 2}-3 d^{10} 4 p^{2} P_{3 / 2}$ & 324.8 & 30783.7 & - & $-680(15 \%)$ & MCDF [61] \\
\hline $\mathrm{Ga}$ & $(Z=31)$ & $4 p^{2} P_{3 / 2}-5 s^{2} S_{1 / 2}$ & 417.3 & 23962.3 & $-431(40 \%)$ & $400(15 \%)$ & MCDF [62] \\
\hline $\mathrm{Y}^{+}$ & $(Z=39)$ & $5 s^{2}{ }^{1} S_{0}-4 d 5 p{ }^{1} P_{1}$ & 363.4 & 27516.7 & $\begin{array}{c}1789^{\mathrm{a}} \\
1318(15 \%)\end{array}$ & $\begin{array}{c}-3181^{\mathrm{a}} \\
-3212(10 \%)\end{array}$ & $\begin{array}{l}\text { semi-emp. [19] } \\
\text { MCDF, this w. }\end{array}$ \\
\hline & & $4 d 5 s^{3} D_{2}-4 d 5 p^{3} P_{1}$ & 321.8 & 31079.0 & $124(15 \%)$ & $-1152(10 \%)$ & MCDF, this w. \\
\hline $\mathrm{Y}^{2+}$ & $(Z=39)$ & $5 s^{2} S_{1 / 2}-5 p^{2} P_{3 / 2}$ & 281.8 & 35487.8 & - & - & - \\
\hline $\mathrm{Nb}^{+}$ & $(Z=41)$ & $4 d^{3} 5 s^{5} F_{1}-4 d^{3} 5 p^{5} F_{1}$ & 290.9 & 34375.0 & $716^{\mathrm{a}}$ & $-2430^{\mathrm{a}}$ & semi-emp. [27] \\
\hline $\mathrm{Ta}^{+}$ & $(Z=73)$ & $6 s^{2}{ }^{3} P_{1}-* J=2^{c}$ & 301.3 & 33184.8 & - & - & - \\
\hline $\mathrm{Os}^{-}$ & $(Z=76)$ & $5 d^{7} 6 s^{2}{ }^{4} F_{9 / 2}-5 d^{6} 6 s^{2} 6 p^{6} D_{9 / 2}$ & 1162.7 & 8600.3 & $\begin{array}{c}2500 \pm 12600 \\
4000(40 \%)\end{array}$ & $\begin{array}{c}16200 \pm 9900 \\
12300(25 \%)\end{array}$ & $\begin{array}{l}\text { Exp. [63] } \\
\text { MCDF }\end{array}$ \\
\hline Po & $(Z=84)$ & $\begin{array}{l}6 p^{3} 7 s^{5} S_{2}-6 p^{3} 7 p^{5} P_{2} \\
6 p^{4}{ }^{3} P_{2}-6 p^{3} 7 s{ }^{5} S_{2}\end{array}$ & $\begin{array}{l}843.4 \\
255.8\end{array}$ & $\begin{array}{l}11853.7 \\
39081.2\end{array}$ & $\begin{array}{l}-311(25 \%) \\
51(25 \%)\end{array}$ & $\begin{array}{l}-12786(15 \%) \\
28663(15 \%)\end{array}$ & $\begin{array}{c}\text { MCDF } \\
{[30]}\end{array}$ \\
\hline
\end{tabular}

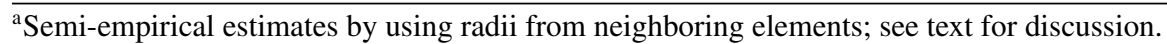

${ }^{\mathrm{b}}$ Experiments have been performed for the naturally occurring isotopes by Kellerbauer et al. [63].

${ }^{\mathrm{c}}$ Upper state is second-order perturbed but not to the extent of the studied transitions from the ground state [64].
} 
references), additional computations have been performed for two low-lying transitions in yttrium. In these computations, and similarly to gallium as discussed in Sec. V B, the following four computational models were considered for the construction of the wave functions from Eq. (12). Model A: Averaged Dirac-Fock (DF) calculations for just the reference configurations, including the Breit interaction into the Hamiltonian matrix in addition to the mass- and field-shift contributions as discussed above. The radial orbitals calculated in this model are kept frozen in all further computations. Model B: Extended optimized level (EOL) calculations, carried out together for the lower and upper levels of the given transition, and including single and double (SD) virtual excitations from the reference configuration into a full layer of correlation orbitals $\{(n+1) s,(n+1) p,(n+1) d,(n+1) f\}$. Here, $n$ refers to the maximum principal quantum number that is occupied in usually the upper level of the transition. Model $C$ : The same as model B but by incorporating, in addition, the core-polarization from all occupied $1 s, \ldots, n l j$ subshells (by means of single excitations) and by reoptimizing the radial orbitals of the $(n+1)$ correlation layer from above. Model D: EOL calculations for the two levels of interest including core-core excitations from a selected set of subvalence shells and SD excitations into an additional $(n+2)$ layer of correlation orbitals.

While these four approaches may result in quite sizable expansions already, no further attempt has been made to push the wave-function expansions to its present-day limits or to establish an independent variational procedure for each level of interest. A further systematic enlargement of the wavefunction expansion is usually possible only for a single element and transition at a given time and might be considered in the future.

Similar computations, including at least model C, have been carried out for all elements and transitions for which MCDF results are displayed in Table I $[69,72]$.

\section{CASE STUDIES AND DISCUSSION}

Those calculations as described in the previous section have recently been applied to a wide range of elements in answer to the call from the radioactive ion beam physics community. Their challenges and implications are described below. Examples are given for elements from across the nuclear chart, in an order which roughly indicates the increasing need for theoretical calculations. As a general rule, for the heaviest elements, the field shift dominates over the mass shift (which is often neglected). In the medium mass $(A \sim 100)$ region this is not the case, but neighboring isotopic chains may be used as a guide to the general trend of the radii (when no alternative is available) which tend to be dominated by macroscopic behavior. Neither of these simplified approaches are applicable to lighter elements. On the other hand, if two stable isotopes exist for the element under study, a nonoptical measurement of $\delta\left\langle r^{2}\right\rangle$ may be used to infer the mass shift, leaving only the field shift to be calculated. It seems clear that this task is far simpler since the nuclear potential of an extended nucleus is usually under much better control than the correlation effects, upon which the specific mass shift depends. For elements with only a single isotope, calculation of the mass shift is required and with no (nonoptical) data to provide an independent check. Elements of the highest $Z$ are studied where no stable or even naturally occurring isotopes exist, and atomic data of any type become increasingly scarce.

\section{A. Yttrium and niobium}

In the $A \sim 100$ region of the nuclear chart, a sudden onset of nuclear deformation occurs at $N \sim 60$, which appears centered around the yttrium chain [73]. A corresponding increase in $\left\langle r^{2}\right\rangle$ is seen, and the isotope shifts have been recently studied in yttrium [19] and niobium [27], requiring the evaluation of the atomic factors for the extraction of this nuclear observable of interest. Many stable isotopes exist in neighboring chains, such as zirconium [18] and molybdenum [74], allowing these radii to be established using nonoptical techniques. This then allows the calibration of $F$ and $M$ for the studied optical transition using a King plot [1] and the determination of $\delta\left\langle r^{2}\right\rangle$ from all measured isotope shifts. However, for yttrium and niobium, only one stable isotope exists in each case, and therefore no independent measurements of $\delta\left\langle r^{2}\right\rangle$ are available. Reliable values of $F$ and $M$ are essential for determining the overall gradient of the radii, how this changes with $Z$, and the consequences for determining the changing softness of the deformation over the shape transition, via Eq. (1).

In the absence of any calibration for the atomic factors of yttrium and niobium, an assumption was made that the systematics of the charge radii, close to stability, varied little with $Z$. For niobium, existing $\delta\left\langle r^{2}\right\rangle$ values from the neighboring zirconium chain were used (for the same neutron number pairs) as if they were nonoptical measurements of niobium [27]. A King plot was formed from the measured isotope shifts in niobium, $v^{N, N^{\prime}}$, and the $\delta\left\langle r^{2}\right\rangle^{N, N^{\prime}}$ values of zirconium (using only those isotopes away from the anomalous $N=60$ region of structural change). This produces a fairly linear plot [73], but the confidence in the extracted parameters depends on the validity of the approximation made above.

For yttrium [19], a modified King plot was formed incorporating measurements for $N=47-59$. Isotopes from beyond the structural change at $N=59$ reduced the linearity of the plot. An extended approach was used, whereby in addition to using the zirconium radii, the process was repeated using the radii of krypton, rubidium, and strontium. In each case, the atomic factors were slightly different and are shown in Fig. 2. Nevertheless, for the studied $363 \mathrm{~nm} 5 s^{2}{ }^{1} S_{0}-5 s 5 p{ }^{1} P_{1}$ ionic ground-state transition, estimates of $F_{363}=-3181 \mathrm{MHz} / \mathrm{fm}^{2}$ and $M_{363}=+1789 \mathrm{GHz}$ amu were made and used to extract the mean-square charge radii [19]. These are shown in Fig. 3. Selected radii were later remeasured using a second transition [75].

As part of this work, theoretical estimates of the atomic factors were produced. Following the computational procedure as described above, systematically enlarged MCDF calculations have been performed for the two transitions $5 s^{2}{ }^{1} S_{0}-5 s 5 p{ }^{1} P_{1}$ and $4 d 5 s{ }^{3} D_{2}-4 d 5 p{ }^{3} P_{1}$ in yttrium. For describing these transitions, we have chosen the reference configurations $4 d^{2}, 4 d 5 s$, and $5 s^{2}$ for the ${ }^{1} S_{0}$ and ${ }^{3} D_{2}$ lower levels, and $4 d 5 p, 5 s 5 p$ for the ${ }^{1,3} P_{1}$ upper levels. In practice, also the $4 p^{6}$ closed shell were added to the valence electrons as excitations of a $4 p$ (sub) valence electron also contribute 


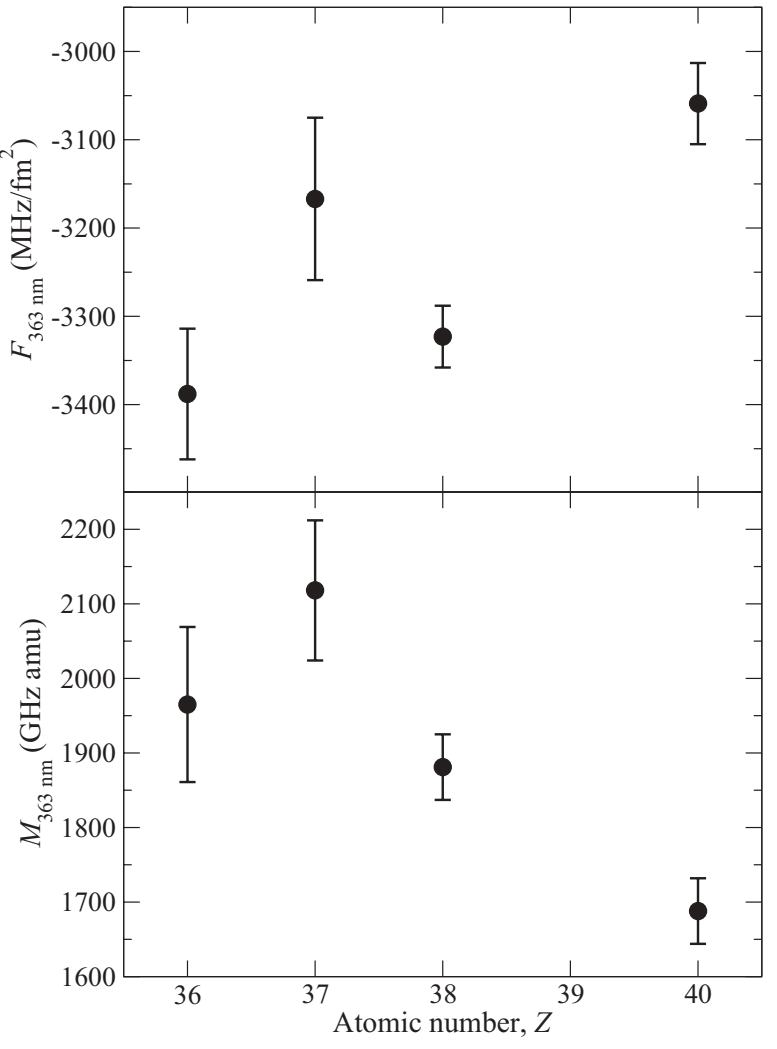

FIG. 2. Estimates of the atomic factors for the $363 \mathrm{~nm} J=$ $0 \rightarrow J=1$ ionic transition in yttrium. A King plot was formed from the measured isotope shifts in yttrium $\delta v^{N, N^{\prime}}$ together with $\delta\left\langle r^{2}\right\rangle^{N, N^{\prime}}$ values for various neighboring elements. Isotopes with neutron numbers in the range $N=47-59$ were used.

to the low-lying level structure of $\mathrm{Y}^{+}$ions. By performing various computations with an increasing number of CSF, $n_{c}$, a quite sizable change of the $M$ and $F$ parameters were caused

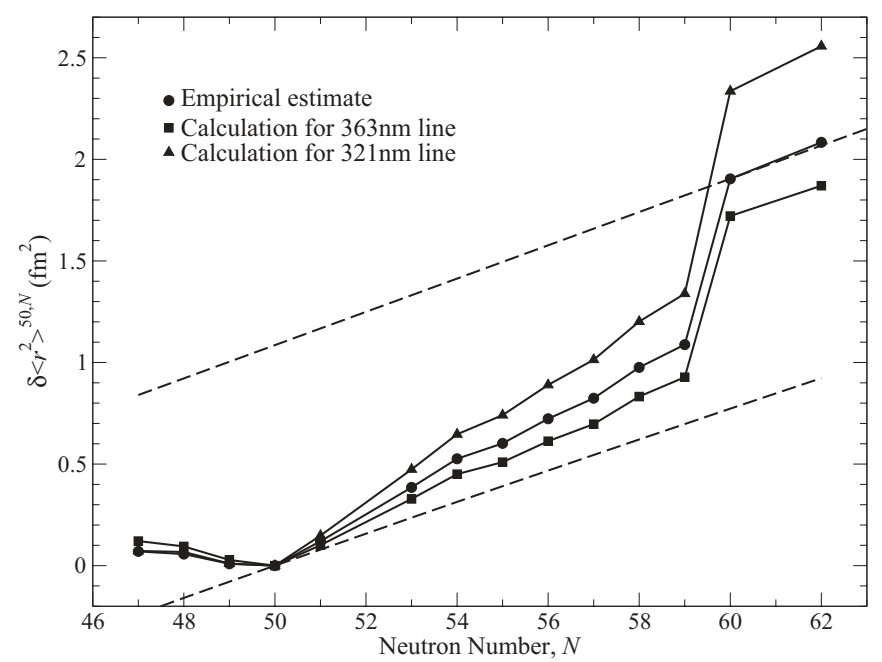

FIG. 3. Mean-square charge radii of yttrium isotopes, extracted from IS measurements using (a) an empirical estimate of the atomic factors, (b) theoretical calculation of the atomic factors for the $363 \mathrm{~nm}$ ionic transition, and (c) theoretical calculation of the atomic factors for the $321 \mathrm{~nm}$ ionic transition.
TABLE II. Theoretical estimates for two ionic transitions in yttrium, the $363 \mathrm{~nm} 5 s^{2}{ }^{1} S_{0}$ (ground state) $\rightarrow 4 d 5 p{ }^{1} P_{1}$ $\left(27516.691 \mathrm{~cm}^{-1}\right)$ and $321 \mathrm{~nm} 4 d 5 s^{3} D_{2}\left(1045 \mathrm{~cm}^{-1}\right) \rightarrow 4 d 5 p^{3} P_{1}$ (32 $124 \mathrm{~cm}^{-1}$ ) lines. The field shift factor $F$, specific mass shift factor $M_{\mathrm{SMS}}$, number of CSF $n_{c}$ and are shown for the four computational models as explained in the text above. The normal mass shift factor $M_{\mathrm{NMS}}$ was calculated to be 453 and $511 \mathrm{GHz}$ amu for the two transitions, respectively.

\begin{tabular}{lcccc}
\hline \hline Transition & $\begin{array}{c}\text { Comput. } \\
\text { model }\end{array}$ & $n_{c}$ & $\begin{array}{c}M_{\mathrm{SMS}} \\
{[\mathrm{GHz} \text { amu }]}\end{array}$ & $\begin{array}{c}F \\
{\left[\mathrm{MHz} / \mathrm{fm}^{2}\right]}\end{array}$ \\
\hline $363 \mathrm{~nm}$ & $\mathrm{~A}$ & 30 & 1088 & -2344 \\
& $\mathrm{~B}$ & 11593 & 1009 & -2982 \\
& $\mathrm{C}$ & 14245 & 772 & -3272 \\
$321 \mathrm{~nm}$ & $\mathrm{D}$ & $64962^{\mathrm{a}}$ & 865 & -3212 \\
& $\mathrm{~A}$ & 30 & -1661 & -1336 \\
& $\mathrm{~B}$ & 19518 & -413 & -1162 \\
& $\mathrm{C}$ & 23286 & -305 & -1090 \\
& $\mathrm{D}$ & $108176^{\mathrm{a}}$ & -387 & -1152 \\
\hline \hline
\end{tabular}

Including core-core correlations only from the $4 s$ and $4 p$ shells.

especially by the core-polarization contributions, although restricted core-core correlations still give rise to noticeable shifts. Because of the open $4 d, 5 s$, and $5 p$ shells, however, no clear convergence of the mass shift could be shown yet. From the variations of the $M$ and $F$ parameters in Table II (and further computations not shown here), we expect an uncertainty of about $25 \%$ for the specific mass shift and $15 \%$ for the field shift parameters. Due to the normal mass shift of $\sim 500 \mathrm{GHz}$ amu, the uncertainties on the total mass shift parameter reduce to about $15 \%$ for both the 363 and $321 \mathrm{~nm}$ transitions of the $\mathrm{Y}^{+}$ion.

The MCDF values for the $363 \mathrm{~nm}$ transition were used to calculate the mean-square charge radii which are added to Fig. 3. Compared with the empirically derived values used in Ref. [19], the calculated field factor of $-3212 \mathrm{MHz} / \mathrm{fm}^{2}$ is similar, but the total mass shift factor $+1318 \mathrm{GHz}$ amu is smaller. On the neutron-rich side, this results in radii which are smaller than those previously reported. However, in Ref. [19], estimates of the mean-square charge radii were produced, via Eq. (1), using the droplet model combined with the deformation deduced from the quadrupole moments. Although themselves subject to a systematic error, those values are expected to provide an underestimate [19] of the mean-square charge radius, but are higher than the values calculated here.

In the later experiment, selected isotope shifts were remeasured on the $321 \mathrm{~nm} 4 d 5 s{ }^{3} D_{2}-4 d 5 p{ }^{3} P_{1}$ ionic transition from a metastable state [75]. A King plot of the modified $321 \mathrm{~nm}$ isotope shifts ( $y$ axis) versus the modified $363 \mathrm{~nm}$ isotope shifts ( $x$ axis) was performed, yielding a gradient of $+0.44178(98)$ and intercept of $-676668(1958) \mathrm{MHz}$. This provides a useful check of the theoretical calculations if these are made for both transitions.

Calculations were therefore performed as part of this work for the $321 \mathrm{~nm}$ transition. In this case the field factor $F_{321}=$ $-1152 \mathrm{MHz} / \mathrm{fm}^{2}$ is smaller than the empirically derived value $\left(F_{321}=-1405 \mathrm{MHz} / \mathrm{fm}^{2}\right.$ and $M_{321}=+114 \mathrm{GHz}$ amu [75] $)$ whereas $M_{321}=+124 \mathrm{GHz}$ amu is similar. Charge radii calculated from the $321 \mathrm{~nm}$ isotope shifts and the MCDF 
TABLE III. The same as Table II but for the $417 \mathrm{~nm} 4 p^{2} P_{3 / 2}-$ $5 s{ }^{2} S_{1 / 2}$ line in neutral gallium. The normal mass shift factor $M_{\mathrm{NMS}}$ was calculated to be $394 \mathrm{GHz}$ amu.

\begin{tabular}{lcccc}
\hline \hline Transition & $\begin{array}{c}\text { Comput. } \\
\text { model }\end{array}$ & $N_{\mathrm{CSF}}$ & $\begin{array}{c}M_{\mathrm{SMS}} \\
{[\mathrm{GHz} \text { amu }]}\end{array}$ & $\begin{array}{c}F \\
{\left[\mathrm{MHz} / \mathrm{fm}^{2}\right]}\end{array}$ \\
\hline $417 \mathrm{~nm}$ & $\mathrm{~A}$ & 3 & -1332 & 169 \\
& $\mathrm{~B}$ & 3739 & -1036 & 383 \\
& $\mathrm{C}$ & $3873^{\mathrm{a}}$ & -973 & 407 \\
& $\mathrm{D}$ & $63128^{\mathrm{b}}$ & -825 & 400 \\
\hline \hline
\end{tabular}

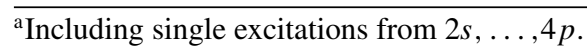

${ }^{\mathrm{b}}$ Including also some SD excitations into a third but incomplete correlation layer.

atomic factors are plotted in Fig. 3. Moreover, the theoretical values for the 321-nm line can be used in conjunction with the King plot to calculate the equivalent factors for the 363-nm line $\left(F_{363}=-2608 \mathrm{MHz} / \mathrm{fm}^{2}, M_{363}=+1812 \mathrm{GHz} \mathrm{amu}\right)$, and therefore show the radii for all of the isotopes. In this case, the radii of the neutron-rich isotopes are much larger than the empirically derived values and than the regional nuclear systematics would suggest. It may therefore be concluded from the King plot that the theoretical calculations for the two transitions are not fully consistent with each other.

\section{B. Gallium and copper}

Isotope shift measurements have recently been performed for gallium [62] and copper [61] isotopes. These studies were motivated by the reordering of the nuclear energy levels among the neutron-rich isotopes that occur due to the monopole component of the nucleon-nucleon force [28]. Moreover, the formation of a diffuse proton structure (skin) in the neutrondeficient gallium isotopes had been proposed to explain the apparent increase in matter radius with decreasing neutron number. If indeed present, this would have a significant effect on the charge radius. For both elements, however, only two stable isotopes exist. Calculations for the gallium chain are discussed below.

For gallium, IS measurements were performed for isotopes in the range ${ }^{63-82} \mathrm{Ga}$ [62]. Mean-square charge radii measurements had only previously been made for the stable ${ }^{69,71} \mathrm{Ga}$ isotopes using muonic atoms, yielding $\delta\left\langle r^{2}\right\rangle^{71,69}=$ $-0.12(2) \mathrm{fm}^{2}$ [54]. Though this single value can be used to provide a consistency check for theoretical calculations, it is insufficient to provide a full calibration.

Similar computations as for yttrium have been performed for the experimentally studied $4 p^{2} P_{3 / 2}-5 s^{2} S_{1 / 2}$ transition in atomic gallium (see Table III). Although this transition refers nominally to a rather simple single electron excitation outside of a closed core, the (nearly degenerate) $4 s$ shell affects this transition strongly and suggests applying the $4 s^{2} 4 p$ and $4 s^{2} 5 s$ reference configuration. Starting from the DF computation with these reference configurations, a large effect on the mass and field shift parameters $M$ and $F$ is observed by including SD valence excitations into the $6 l$ layer for the levels with total angular momenta and parities, $J^{P}=1 / 2^{+}$and $3 / 2^{-}$. Again, further core-polarization and core-core correlations, including also excitations from the $3 d$ shell, still give rise to sizable changes, although some of the correlation contributions tend to cancel each other. At present, however, attention has been paid more systematically only to the core-valence and corepolarization effects by including SD excitations into a second and part of a third correlation layer. From these computations, we estimate an overall uncertainty of $15 \%$ for the $F$ and $40 \%$ for the $M$ parameter of the $4 p^{2} P_{3 / 2}-5 s^{2} S_{1 / 2}$ transition.

Using the measured IS $\delta v^{71,69}=+40(4) \mathrm{MHz}$ [62], the calculated atomic factors $\left[F_{417}=+400(60) \mathrm{MHz} / \mathrm{fm}^{2}\right.$, $M_{417}=-431(170) \mathrm{GHz}$ amu] yield a difference in radius of $\delta\left\langle r^{2}\right\rangle^{71,69}=-0.34 \mathrm{fm}^{2}$, significantly larger than the nonoptical data. Of the two factors, the field factor appears to stabilize more readily than the mass shift factor. Assuming the inconsistency is therefore due to the second of these, and the field factor and muonic data (although nuclear model dependent) are correct, we may alternatively calculate the mass shift factor to be $M_{417}=-216 \mathrm{GHz}$ amu. Although many of the qualitative details of the extracted radii remain unchanged, an uncertainty in the mass shift parameter $M$ causes a tilting of $\delta\left\langle r^{2}\right\rangle$ about the reference point and prevents the general gradient from becoming clear.

\section{Scandium and manganese}

Studies between the $Z=20$ and $Z=28$ proton shell closures have recently included IS measurements in scandium [10] and manganese [60]. As with yttrium and niobium, these elements each have only a single stable isotope and nonoptical measurements of the absolute radius have only been made for these isotopes, with no $\delta\left\langle r^{2}\right\rangle$ available. Unlike the heavier yttrium and niobium nuclei, microscopic phenomena are more apparent in the radii and less correlation is seen between neighboring elements. This leaves any evaluation of $\delta\left\langle r^{2}\right\rangle$ entirely dependent on theoretical calculations.

Anomalous charge radii provided the primary motivation for the scandium experiments [10]. Whereas the radii generally increase between shell closures, those in calcium form a parabolic trend between $N=20$ and $N=28$ [76]. For this reason, ${ }^{48} \mathrm{Ca}$ has a very similar radius to ${ }^{40} \mathrm{Ca}$. In titanium, in contrast, the radii were seen to increase as the neutron number decreased below midshell. Scandium lies therefore in the critical position between these two chains and the accurate determination of the evolution of the radii is required. MCDF calculations of the atomic factors were performed and described in detail by the authors of Ref. [10]; they give rise to radii with a titanium-like increase as the neutron number decreases. Nuclear shell model calculations produced estimates more similar to the calcium radii, but this would require the mass shift factor to lie beyond the quoted error [10]. Further experiments are envisaged, which will take the IS measurements past the $N=28$ shell closure [77].

For manganese radioisotopes, laser spectroscopy has begun with an initial study of ${ }^{50-56} \mathrm{Mn}$ [60] and are the first of such measurements in the neighborhood. For these isotopes, the spectroscopy was performed on the $3 d^{5} 4 s{ }^{5} S_{2}$ $\left(9472.970 \mathrm{~cm}^{-1}\right) \rightarrow 3 d^{5} 4 p^{5} P_{3}\left(43370.510 \mathrm{~cm}^{-1}\right)$ transition at $295 \mathrm{~nm}$. These measurements crossed the neutron number $N=28$, and revealed a minimum in $\delta\left\langle r^{2}\right\rangle$ which seems a signature of a neutron shell closure. Further spectroscopy is planned to look for subshell effects at $N=32$ and $N=34$, 


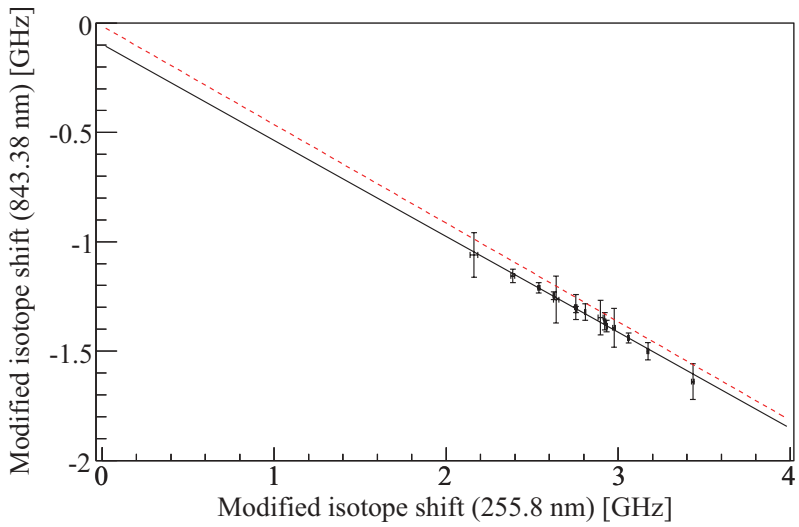

FIG. 4. (Color online) Modified King plot between the two transitions of Po-I studied with laser spectroscopy [30,81]. The straight line is the best fit through the data points; the dashed line is the present calculations.

and to investigate an onset of collectivity, suggested to occur towards $N=40$.

\section{Translead nuclei}

The elements around lead are of great interest to nuclear physics for the richness of their structure: magic shell closures at $Z=82$ and $N=126$, shape coexistence in the neutrondeficient nuclei $[78,79]$, as well as octupole deformation in the neutron-rich nuclei [80]. These phenomena are directly reflected on the shape of the nuclear ground state and have prompted extensive laser spectroscopic studies [1].

The elements with $Z>82$ have only one (near)stable isotope (bismuth, $Z=83$ ), while all other nuclides are radioactive. Most of those isotopes are also not naturally occurring and have to be produced in the laboratory. As a consequence, the knowledge on their atomic structure is limited. Moreover, there are no existing charge radii that can be used for a King plot analysis.

As with the yttrium data, it is possible to assert the accuracy of the atomic calculations to some extent, by comparing the modified isotope shifts of two different transitions using a King plot. This approach was used in the study of the polonium isotopes [30]. The modified King plot is shown in Fig. 4. As seen from this figure, the MCDF dotted line is parallel to the best fit through the data points. We therefore conclude that the ratio $F_{256} / F_{843}$ is accurate, giving high confidence in the independent field shift factors. The difference in the $y$ intercepts shows, however, the discrepancy in the mass shift factors. This corresponds to the behavior of the respective parameters during the calculations: while the field shift factors $F$ converge reasonably fast, the mass shift factors $M$ are more sensitive to the details of the calculations. The modified King plot analysis was used to determine the systematic uncertainty arising from the mass shift. This systematic uncertainty is comparable to the statistical uncertainty and does not affect the nuclear physics conclusions.

In the case of astatine, the situation is even more complicated, as only two atomic transitions were observed in absorption spectroscopy [82]. A recent online campaign of studies at radioactive ion beam facilities [83] has permitted the discovery of many additional transitions. The search for those transitions and their interpretation has been greatly improved by the input from theoretical calculations. New ionization schemes have been developed and the ionization potential has been determined with great accuracy, and compared with the atomic calculations. A campaign for the measurement of the $\delta\left\langle r^{2}\right\rangle$ is proposed and will require the electronic factor and specific mass shift parameters to be calculated.

Finally, in the bismuth isotopes, a large data set of $\delta v$ is available [84] but no $\delta\left\langle r^{2}\right\rangle$ could be extracted. Alternative empirical approaches have been used to interpret the nuclear effects on the $\delta v$ but new atomic calculations are required to finalize the analysis.

Another experimental program will study the anomalous reduction in mean-square charge radius seen for isomeric states with a large number of unpaired nucleons [36]. A wealth of such states exist in ${ }^{178-182}$ Ta and these studies will significantly add to the quantitative information available. Furthermore, the study of these isomers in near-spherical bismuth nuclei will allow the origin of the effect (be it rigidity of deformation or diffuseness of the nuclear surface) to be investigated [85]. The same effect may be responsible for the odd- $N$ or even- $N$ staggering of $\delta\left\langle r^{2}\right\rangle$, seen across the nuclear chart, but still poorly understood from a qualitative point of view.

\section{E. Very heavy and superheavy nuclei}

The properties of superheavy nuclei are a road map towards the limit of nuclear existence. They also offer an uncharted ground where atomic and nuclear models can be tested, but more often than not, studies on these elements suffer from their exotic nature and their absence from nature. As these elements may only be produced in the laboratory and studied in very limited quantities, almost no information is available on their atomic structure.

In an effort to reach towards the transfermic nuclei, a first step is to bridge the gap from the stable elements to uranium. An international effort is currently underway at radioactive ion beam facilities to study the nuclei from actinium to fermium [1]. New facilities are also under construction where more systematic studies will be performed [86]. Those studies will eventually require atomic calculations, similarly as was done for the case of astatine mentioned above, in the search for atomic levels and subsequently in the measurement campaigns on $\delta\left\langle r^{2}\right\rangle$.

For most (super) heavy elements, accurate IS calculations still remain a challenge. Although a reasonable agreement between experimental and calculated IS parameters was found for singly charged $\mathrm{Os}^{-}$ions [63] and Po atoms [30], additional computations are needed to shed insight into the effects of a (nearly) open $5 f$ shell for the actinides and beyond. At present, the MCDF method seems to be most suitable for performing such computations, and especially for the elements for which only limited or no atomic data are available yet.

For fermium $(Z=100)$, for example, first successful measurements on the low-lying level structure were recently based on prior MCDF computations [87]. Using a sample of $2.7 \times 10^{10}$ atoms of the isotope ${ }^{255} \mathrm{Fm}$ with a half-life of 20.1 hours only, two atomic levels were found at wave numbers $25099.8 \pm 0.2$ and $25111.8 \pm 0.2 \mathrm{~cm}^{-1}$ and assigned as 
$5 f^{12} 7 s 7 p^{5} I_{6}^{o}$ and $5 f^{12} 7 s 7 p^{5} G_{5}^{o}$, with a remarkable agreement between the experiment and calculations. Moreover, partial lifetimes were determined from the saturation characteristics and helped assign the corresponding terms, by comparing the transition probabilities. While a similar relative accuracy as for the level energies cannot be expected in general for most other properties, these and more recent computations clearly show the capabilities and potential of the MCDF method for complex shell structures [88].

\section{SUMMARY AND CONCLUSION}

As discussed above, the quest for accurate IS calculations and parameters cannot be separated from the shell structure and electronic transitions that are utilized in laser spectroscopy. Simple shell structures with just one or two electrons outside of closed shells and some higher charge state of the ions typically increase the stability of the electrons and, hence, may help reduce the computational effort (for instance, the size of the wave functions to obtain a required accuracy). However, this applies only as long as QED effects are negligible or remain moderate [89]. Moreover, $s-p$ and $p-s$ transitions are generally simpler than transitions with open $d$ and $f$ shells; these rather general rules may suggest to explore laser transitions in the future where, as far as lasers are available, the constraints from atomic structure theory are taken into account in the preparation and setup of new experiments.

From an experimental point of view, transitions are normally selected on the basis of signal counts per ion sensitivity. Also, they are selected for the ability to extract the nuclear properties with precision. For example, each hyperfine structure needs to (i) be large enough to be resolvable, (ii) posses a level with a large electronic field gradient for an accurate determination of the quadrupole moment, and (iii) involve states with $J>1 / 2$ for the determination of the nuclear spin. Measuring a transition with relatively large isotope shifts (but experimental errors still much the same) would increase the precision of the extracted $\delta\left\langle r^{2}\right\rangle$, but this advantage is lost if the calculation of the field factor is less certain. Optical transitions are generally chosen to be from the ground state for reasons of natural population. However, the method of in-cooler optical pumping enables transitions from low-lying ionic metastable states to be studied [27], while long-lived atomic metastable states may be quasiresonantly populated in the charge exchange process. Experimental beam time is extremely valuable, but even if a transition is spectroscopically weaker, a selected remeasurement of just a few of the more intensely produced isotopes would enable the consistency of the calculated atomic factors to be assessed via a King plot (as for Y, Po).

Several elements have too few naturally occurring isotopes for an experimental calibration of the atomic factors (and even these values are nuclear model dependent). Only some examples have been mentioned here, where radioactive studies have recently been made or are still in progress. In the case of manganese, yttrium, tantalum, polonium, and astatine, further experiments are planned to take place in the near future. Atomic factor calculations are critical for the reliable extraction of nuclear mean-square charge radii and the conclusions which are drawn from them. For manganese, studies will be extended to more neutron-rich isotopes, looking for the predicted new subshell closures at $N=32$ and $N=34$ and an onset of collectivity around $N=40$. Nuclear $\delta\left\langle r^{2}\right\rangle$ values are a key indicator of these phenomena. Several existing measurements will be repeated using a transition in the neutral atom (see Table I) and the atomic factor calculations compared with those for the ionic transition. High deformation in the vicinity of ${ }^{80} \mathrm{Zr}$ will be probed via the exploration of neutron-deficient yttrium isotopes. A useful insight into the nature of the deformation (its rigidity or softness) can be obtained through a quantitative comparison with quadrupole-moment-derived estimates. Measurements of nuclear isomeric and ground states of just a few tantalum isotopes will greatly increase the limited data available on multi-quasiparticle isomers. It is hoped that this will then encourage attempts to model the anomalous reductions seen in the isomeric charge radii. These are only a few examples of the experiments that will be carried out within the next couple of years alone, at ISOLDE, TRIUMF, and JYFL, respectively. In each case, a reliable calibration of the mass shift (as well as the field shift) is essential for a full analysis of the experimental data.

We hope that the present work and discussion will stimulate improved IS calculations, either by using enlarged MCDF wave functions [70,71], many-body perturbation calculations [58,90], or even a more sophisticated QED treatment [91]. Especially for heavy and superheavy elements, only a systematic treatment of all major relativistic, QED, and correlation effects will enable one to extract useful information from laser spectroscopy about the structure, interactions, and charge distributions of radioactive nuclei.

\section{ACKNOWLEDGMENTS}

B.C. was supported by the UK Science and Technology Facilities Council (STFC).
[1] B. Cheal and K. T. Flanagan, J. Phys. G 37, 113101 (2010).

[2] H. J. Kluge et al., Hyperfine Interact. 196, 295 (2010).

[3] E. W. Otten, in Treatise on Heavy-Ion Science, edited by D. A. Bromley, (Plenum Press, New York, 1989), Vol. 8, p. 517.

[4] J. Billowes and P. Campbell, J. Phys. G 21, 707 (1995).

[5] H. J. Kluge and W. Nörtershäuser, Spectrochim. Acta, Part B 58, 1031 (2003).
[6] W. D. Myers and K.-H. Schmidt, Nucl. Phys. A 410, 61 (1983).

[7] D. Berdichevsky and F. Tondeur, Z. Phys. A 322, 141 (1985).

[8] P. Möller, J. R. Nix, W. D. Myers, and W. J. Swiatecki, At. Data Nucl. Data Tables 59, 185 (1995).

[9] E. Caurier et al., Phys. Lett. B 522, 240 (2001).

[10] M. Avgoulea et al., J. Phys. G 38, 025104 (2011). 
[11] M. Bender, P.-H. Heenen, and P.-G. Reinhard, Rev. Mod. Phys. 75, 121 (2003).

[12] G. A. Lalazissis, S. Raman, and P. Ring, At. Data Nucl. Data Tables 71, 1 (1999).

[13] S. Goriely, F. Tondeur, and J. M. Pearson, At. Data Nucl. Data Tables 77, 311 (2001).

[14] R. Rodríguez-Guzmán, P. Sarriguren, L. Robledo, and S. PerezMartin, Phys. Lett. B 691, 202 (2010).

[15] R. Rodriguez-Guzman, P. Sarriguren, and L. M. Robledo, Phys. Rev. C 82, 061302 (2010).

[16] R. Rodriguez-Guzman, P. Sarriguren, and L. M. Robledo, Phys. Rev. C 83, 044307 (2011).

[17] D. T. Yordanov et al., Phys. Rev. Lett. 108, 042504 (2012).

[18] P. Campbell et al., Phys. Rev. Lett. 89, 082501 (2002).

[19] B. Cheal et al., Phys. Lett. B 645, 133 (2007).

[20] W. Nörtershäuser et al., Phys. Rev. Lett. 102, 062503 (2009).

[21] V. A. Dzuba, W. R. Johnson, and M. S. Safronova, Phys. Rev. A 72, 022503 (2005).

[22] E. Mané, A. Voss et al., Phys. Rev. Lett. 107, 212502 (2011).

[23] A. Krieger et al., Phys. Rev. Lett. 108, 142501 (2012).

[24] E. C. Seltzer, Phys. Rev. 188, 1916 (1969).

[25] G. Torbohm, B. Fricke, and A. Rosén, Phys. Rev. A 31, 2038 (1985).

[26] W. H. King, Isotope Shifts in Atomic Spectra (Plenum Press, New York, 1984).

[27] B. Cheal et al., Phys. Rev. Lett. 102, 222501 (2009).

[28] B. Cheal et al., Phys. Rev. Lett. 104, 252502 (2010).

[29] T. E. Cocolios et al., Phys. Rev. Lett. 103, 102501 (2009).

[30] T. E. Cocolios et al., Phys. Rev. Lett. 106, 052503 (2011).

[31] P. Mueller et al., Phys. Rev. Lett. 99, 252501 (2007).

[32] R. Sánchez et al., Phys. Rev. Lett. 96, 033002 (2006).

[33] F. Le Blanc et al., Phys. Rev. C 60, 054310 (1999).

[34] L. Vermeeren, R. E. Silverans, P. Lievens, A. Klein, R. Neugart, C. Schulz, and F. Buchinger, Phys. Rev. Lett. 68, 1679 (1992).

[35] V. N. Fedosseev, Y. Kudryavtsev, and V. I. Mishin, Physica Scripta 85, 058104 (2012).

[36] M. L. Bissell et al., Phys. Lett. B 645, 330 (2007).

[37] V. N. Fedosseev et al., Nucl. Instrum. Methods Phys. Res., Sect. B 266, 4378 (2008).

[38] E. J. Prime et al., Hyperfine Interact. 171, 127 (2006).

[39] Y. Liu et al., Nucl. Instrum. Methods Phys. Res., Sect. B 269, 2771 (2011).

[40] N. Lecesne et al., Rev. Sci. Instrum. 81, 02 A910 (2010).

[41] Y. Kudryavtsev et al., Nucl. Instrum. Methods Phys. Res., Sect. B 179, 412 (2001).

[42] M. Facina et al., Nucl. Instrum. Methods Phys. Res., Sect. B 226, 401 (2004).

[43] I. D. Moore et al., J. Phys. G 31, S1499 (2005).

[44] L. Weissman et al., Phys. Rev. C 65, 024315 (2002).

[45] H. De Witte et al., Phys. Rev. Lett. 98, 112502 (2007).

[46] T. Sonoda et al., Nucl. Instrum. Methods Phys. Res., Sect. B 267, 2918 (2009).

[47] M. D. Seliverstov et al., Eur. Phys. J. A 41, 315 (2009).

[48] H. Backe et al., Hyperfine Interact. 127, 35 (2000).

[49] H. Backe et al., Hyperfine Interact. 162, 3 (2005).

[50] Y. A. Kudriavtsev and V. S. Letokhov, Appl. Phys. B 29, 219 (1982).
[51] J. Billowes, Nucl. Phys. A 682, 206 (2001).

[52] T. J. Procter et al., J. Phys.: Conf. Ser. 381, 012070 (2012).

[53] G. Fricke et al., At. Data Nucl. Data Tables 60, 177 (1995).

[54] G. Fricke and K. Heilig, in SpringerMaterials-The LandoltBörnstein Database, edited by H. Schopper, (Springer-Verlag, Berlin, 2004), Vol. 20.

[55] I. Angeli, At. Data Nucl. Data Tables 87, 185 (2004).

[56] S. A. Blundell et al., J. Phys. B 20, 3663 (1987).

[57] I. I. Tupitsyn, V. M. Shabaev, J. R. CrespoLopez-Urrutia, I. Draganic, R. S. Orts, and J. Ullrich, Phys. Rev. A 68, 022511 (2003).

[58] J. C. Berengut, V. V. Flambaum, and M. G. Kozlov, J. Phys. B 41, 235702 (2008).

[59] E. Gaidamauskas et al., J. Phys. B 44, 175003 (2011).

[60] F. C. Charlwood et al., Phys. Lett. B 690, 346 (2010).

[61] M. L. Bissell et al. (unpublished).

[62] T. J. Procter et al., Phys. Rev. C 86, 034329 (2012).

[63] A. Kellerbauer, C. Canali, A. Fischer, U. Warring, and S. Fritzsche, Phys. Rev. A 84, 062510 (2011).

[64] M. L. Bissell et al., Phys. Rev. C 74, 047301 (2006).

[65] I. P. Grant, in Methods in Computational Chemistry, edited by S. Wilson, (Plenum Press, New York, 1988), Vol. 2, p. 1.

[66] S. Fritzsche, Phys. Scr., T 2002, 37 (2002).

[67] S. Fritzsche, Comput. Phys. Commun. 183, 1525 (2012).

[68] F. Parpia, C. Fischer, and I. Grant, Comput. Phys. Commun. 94, 249 (1996).

[69] S. Fritzsche, J. Electron Spectrosc. Relat. Phenom. 114, 1155 (2001).

[70] P. Jönsson and J. Bieroń, J. Phys. B 43, 074023 (2010).

[71] M. R. Godefroid and C. F. Fischer, Phys. Rev. A 60, R2637 (1999).

[72] S. Fritzsche, C. Fischer, and G. Gaigalas, Comput. Phys. Commun. 148, 103 (2002).

[73] F. C. Charlwood et al., Hyperfine Interact. 196, 143 (2010).

[74] F. C. Charlwood et al., Phys. Lett. B 674, 23 (2009).

[75] K. Baczynska et al., J. Phys. G 37, 105103 (2010).

[76] C. W. P. Palmer, P. E. G. Baird, S. A. Blundell, J. R. Brandenberger, C. J. Foot, D. N. Stacey, and G. K. Woodgate, J. Phys. B 17, 2197 (1984).

[77] B. Cheal and D. H. Forest, Hyperfine Interact. (2012) doi: 10.1007/s10751-012-0621-8.

[78] A. Andreyev et al., Nature (London) 405, 430 (2000).

[79] K. Heyde and J. L. Wood, Rev. Mod. Phys. 83, 1467 (2011).

[80] P. A. Butler and W. Nazarewicz, Rev. Mod. Phys. 68, 349 (1996).

[81] D. Kowalewska et al., Phys. Rev. A 44, R1142 (1991).

[82] R. McLaughlin, J. Opt. Soc. Am. 54, 965 (1964).

[83] S. Rothe et al., (unpublished).

[84] M. R. Pearson et al., J. Phys. G 26, 1829 (2000).

[85] I. D. Moore et al., Hyperfine Interact. 171, 135 (2006).

[86] A. Drouart et al., Nucl. Phys. A 834, 747c (2010).

[87] M. Sewtz et al., Phys. Rev. Lett. 90, 163002 (2003).

[88] S. Fritzsche, C. Z. Dong, F. Koike, and A. Uvarov, Eur. Phys. J. D 45, 107 (2007).

[89] S. Fritzsche, Eur. Phys. J. D 33, 15 (2005).

[90] B. K. Sahoo, J. Phys. B 43, 231001 (2010).

[91] Y. S. Kozhedub, A. V. Volotka, A. N. Artemyev, D. A. Glazov, G. Plunien, V. M. Shabaev, I. I. Tupitsyn, and T. Stohlker, Phys. Rev. A 81, 042513 (2010). 